\title{
Simulated Patient Environment: A Training Tool for Healthcare Professionals in COVID-19 Era
}

\author{
Mangayarkarasi V Babu (D) \\ Mohan Kumar Arumugam ' \\ Dhrubajyoti J Debnath (iD) ${ }^{2}$ \\ 'Department of Microbiology, \\ ${ }^{2}$ Department of Community and Family \\ Medicine, All India Institute of Medical \\ Sciences, Mangalagiri, Andhra Pradesh, \\ India
}

\section{Video abstract}

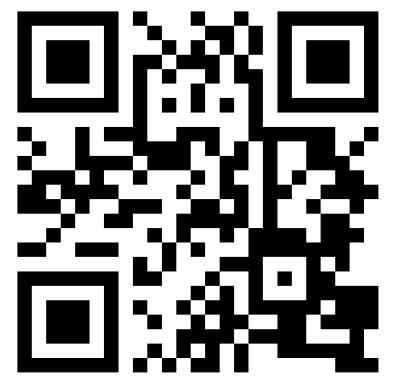

Point your SmartPhone at the code above. If you have a $Q R$ code reader the video abstract will appear. Or use: https://youtu.be/_WZgwpomVHs
Correspondence: Mangayarkarasi V Babu Department of Microbiology, All India Institute of Medical Science, Mangalagiri, Andhra Pradesh, India Tel +9l-98404I 0566

Email mangai.micro@aiimsmangalagiri.edu.in
Background: An in-situ simulation model with a simulated patient environment is a training tool that was implemented for the front-line workers managing COVID-19 respiratory infection outbreak in a hospital of National Importance, situated in southern part of India. The objective of this training tool was to improve the preparedness of health care workers and the control measures to manage any respiratory infection outbreaks. Also, to provide good patient care and patient safety during the sudden outbreak of SARS-CoV-2, the pathogen causing pandemic respiratory infection outbreak worldwide.

Methods: Simulated patient environment is a kind of functional mock drill that was applied to assess and improve the readiness of the healthcare workers of the hospital to identify the patient at the screening area, guide the patient for investigation and inform the public health system during an outbreak of an infection. The faculty of the Department of Microbiology, Community and Family Medicine, and General Medicine were the educators and demonstrators. Fifty healthcare workers from All India Institute of Medical Sciences (AIIMS), Mangalagiri, Ministry of Health and Family Welfare (MOHFW), Government of India, such as doctors, epidemiologists, nurses, laboratory technicians, laboratory attendants, members of infection control team and biomedical waste management team participated in this program. Results: Statistical analysis of the results with pre-intervention and post-intervention assessments and feedback from the participants was done at the end of the program. The difference between the mean of pre-intervention and post-intervention scores in doctors, nurses, and other health care professionals was statistically very highly significant (P-value $<0.001$ ).

Conclusion: The training method was the simulation of management of pandemic respiratory infection outbreaks in the most realistic manner possible to test or evaluate the capability of more than one function in the context of an emergency event. An in-situ simulation is an effective method that is close to real-life scenario, to measure the readiness and effectiveness of an emergency response plan. Hence, it is a useful tool to assess disaster preparedness against any type of outbreak.

Keywords: health care professionals, COVID-19, infection control, pandemics, patient care

\section{Introduction}

The idea behind the application of the training tool, a Simulated Patient - Health Care Workers (HCW) Environment in a hospital, was based on the WHO strategic preparedness and response plan. It was to provide support to all countries to prepare, respond and handle SARS- CoV-2 (COVID-19), the pathogen causing the recent respiratory infection outbreak that started in December 2019.

After the World Health Organization (WHO) declared the pandemic COVID-19 outbreak at the end of the year 2019, our hospital infection control team planned to 
conduct a training program for health care workers on the management of COVID-19 respiratory infection outbreak.

The guidelines given by WHO and Indian Council of Medical Research (ICMR), Ministry of Health and Family Welfare (MOHFW) were mainly to implement the policies, procedures, training, and education, etc. and to take effective administrative infection control measures to prevent the transmission by any health care organization. ${ }^{1-3}$ It required the support of leadership in the health care organization in consultation with hospital management.

Our Hospital, an Institute of National Importance established by the Ministry of Health and Family Welfare, Government of India, is situated in a Southern State of India. Based on the above-said objective, steps were initiated to create awareness through one of the medical education training tools. Simulated patient environment was used as an in-situ simulation model to train health care professionals in COVID-19 outbreak Management in the screening area of the hospital.

Organizational sciences appreciate the need for training in an emergent situation and provide an insight for the advancement of in-situ simulation in health care in terms of more research and implementation. ${ }^{4}$

In-situ simulation is a type of mock drill, which is a relatively new and effective teaching tool to train the front-line workers to manage high-risk disasters such as respiratory infection outbreak. During simulation exercises, health care workers acquire clinical skills by direct practice in comparison to the routine method of teaching and learning. 5

Simulation tools provide an alternative to actual patients. In-situ simulation with the patient - HCW simulation model is conducted in the working environment of the hospital and ideally involves front-line workers team to demonstrate the actual critical and even outbreak management at hospitals. Accordingly, in-situ simulation of outbreak incidents may be a useful, effective method that compares to the available quality assurance procedures in hospitals. ${ }^{6}$ The in-situ simulation model is being used more commonly to evaluate effectiveness of new services and systems in a hospital. ${ }^{7}$

The application of in-situ simulation-based training improves the confidence and the collaborative work skills to respond to an emergency in a real-life clinical setting where multiple disciplines are involved. It helps to considerably improve the safety of patients by promoting the identification of hidden safety hazards. ${ }^{8}$ Research on insitu simulation is growing as a technique to provide continuing medical education to healthcare workers training in real-life clinical scenario. ${ }^{9}$

We aimed to perform a Simulated Patient - Health Care Workers (HCW) Environment, a training tool to improve the preparedness and control measures to be taken for respiratory Infection Outbreak in a hospital. Also, to provide good patient care and patient safety during a sudden outbreak of SARS-CoV-2, the pathogen causing pandemic respiratory infections outbreak worldwide.

\section{Methods}

\section{Sampling Method and Control Group}

To update the knowledge and skills of the doctors, nurses, and other health care workers regarding COVID-19, several training programs were carried out in the Institution regularly. This particular training program was one of the foremost training sessions concerning COVID-19 and involved the doctors, nurses, and other health care workers. They were invited to participate in this study on a scheduled date and time. The institutional ethical committee approval and written consent from the participants were obtained. About 16 doctors, 23 nursing officers, and 11 other health care workers participated in this training session and all of them voluntarily participated in this study. This was a Quasi-experimental study with pre-intervention and post-intervention. There was no control group. No randomization was done.

\section{Study Design}

This was a Quasi-experimental pre-post-intervention study design of In-situ simulation, a training tool for acute respiratory infection outbreak management. The study was performed in screening the COVID-19 suspected patients in the outpatient department of the hospital involving healthcare workers from multiple disciplines. The procedures of simulation training were done in screening area of the outpatient department of the study hospital and it consist of the following:

- Registration of patients to get the patient information by Nursing and Junior doctors' team

- Examination of the patient by the physician

- Nasopharyngeal swab collection for laboratory diagnosis and

- Transportation of sample to the laboratory, by laboratory personnel following strict Infection Control measures and Biomedical waste management measures, etc 
- The community and family health care professional guiding the patient to get complete treatment guidelines.

Data was collected from the participants and analysed for evaluating the effectiveness of the in-situ simulation.

\section{Study Setting}

The in-situ simulation of acute respiratory infection outbreak management in the hospital was conducted in the respiratory outpatient department by the Department of Microbiology in collaboration with the Department of Community and Family Medicine and Department of General Medicine of All India Institute of Medical Sciences (AIIMS), Mangalagiri, Andhra Pradesh, India.

\section{Inclusion Criteria}

- Front-line health care workers working in the screening area for COVID-19 infection

\section{Exclusion Criteria}

- Health care workers not working in the screening area for COVID-19 infection

- Housekeeping and multitask workers were excluded from the study

\section{The in-situ Simulation Program}

To conduct the program, various steps were designed as per National and International guidelines ${ }^{10-13}$ and these guidelines were adhered as given below:

Step 1: Permission was obtained from hospital administrators for scheduling the in-situ simulation training program in the outpatient department of the hospital and the informed verbal consent was obtained from all the participants.

Step 2: Invitations were sent to all hospital staff and media to attend the simulation program to create awareness to the public as well.

Step 3: Briefing in the form of Continuing Medical Education (CME) was carried out to teach the knowledge part, a cognitive skill to the participants on, "Pathogen causing Pandemic Respiratory Infections Outbreak management in hospital". The various guidelines and available registration forms to be filled as per WHO and Ministry of Health and Family Welfare, Government of India were collated and distributed to the participants before the simulation program.

Step 4: Awareness pamphlets for the respiratory infection outbreak scenario were prepared. Some of them were published in our social media handle of Twitter and newspapers. They were also given to the patients and distributed to the public.

Public awareness: https://twitter.com/mangalAiimsAP/ status $/ 1222425723723550720 ? \mathrm{~s}=20$

Health professionals: https://twitter.com/ mangalAiimsAP/status/1222424514354610177?s=20

Hindu newspaper link: https://www.thehindu.com/ todays-paper/tp-national/tp-andhrapradesh/mock-drill-held -on-covid-19/article30876048.ece

Standard Operating Protocol (SOP), written by faculty of Department of Microbiology was distributed to the trainers to demonstrate during acute respiratory infection outbreak. This SOP includes all the available guidelines from websites of national and international competent organizations of the COVID-19 management system. The documents to be filled for the diagnosis and treatment of suspected COVID-19 patients were downloaded and distributed to all the participants.

Step 5: A rehearsal on simulated patients and healthcare workers environment was done and checklists (Supplementary material) were prepared for assessing the completeness of the program.

Step 6: A pre-test was conducted on the day of the simulation program, to identify the baseline knowledge and the attitude of the participating healthcare workers using a questionnaire.

Step 7: In-situ simulation training program of various actions required for acute respiratory infection outbreak management in a hospital was conducted. The program evaluation was done by the qualified observers at each stage of simulation through comprehensive checklists (Supplementary material). After the simulation, the entire group of participants was debriefed and relevant points were discussed and reinforced.

Step 8: In the end, a post-test was conducted to assess the effectiveness of the in-situ simulation program. Post-simulation feedback forms were collected from the participants to analyse their perception of the session.

\section{Statistical Analysis Quantitative Variables}

Pre-Intervention and Post-Intervention scores of health care professionals are the quantitative variables. Paired " $t$ " test was applied to compare the difference between pre-intervention and post-intervention mean scores of doctors, nurses, and other healthcare workers. 
Table I Comparison of Pre-Test and Post-Test Scores

\begin{tabular}{|l|l|l|l|l|l|l|l|l|}
\hline \multirow{2}{*}{ Health Care Professionals } & N & \multicolumn{2}{l|}{ Mean (S.D.) } & \multirow{2}{*}{ t- value } & $\begin{array}{l}\text { Effect } \\
\text { Size }\end{array}$ & $\begin{array}{l}\text { 95\% Cl of Effect } \\
\text { Size }\end{array}$ & P-value \\
\cline { 2 - 9 } & Pre-Test & Post-Test & Pre-Test & Post-Test & & & & \\
\hline Doctors \& Epidemiologists & 16 & 16 & $33.44(12.5)$ & $47.5(8.2)$ & -5.05 & -14.06 & -20 to -8.1 & 0.000 \\
\hline Nurses & 23 & 23 & $38.7(8.3)$ & $49.35(5.3)$ & -11.1 & -10.65 & -12.6 to -8.7 & 0.000 \\
\hline Other Health care workers & $\mathrm{II}$ & $\mathrm{II}$ & $\mathrm{II} .45(7.9)$ & $35.45(8.8)$ & -7.42 & -20 & -26.01 to -13.9 & 0.000 \\
\hline
\end{tabular}

\section{Results}

The pre-intervention and post-intervention scores of doctors, nurses, and other healthcare workers followed probability distribution, and the two groups under comparison were dependent on each other. Therefore, a Paired " $\mathrm{t}$ " test was applied to compare the difference between the 2 means of doctors, nurses, and other healthcare workers. P-value and effect size are mentioned in Table 1. The data were entered in Microsoft Excel. Primer of biostatistics software (Glantz, Stanton A. Primer of Biostatistics. 5th Edition. Publisher McGraw-Hill) was used to analyse the data.

Statistically very highly significant differences were noted between the mean pre-test and post-test scores in doctors, nurses, and other health care professionals (Table 1).

\section{Outcome}

The in-situ simulation training program for COVID-19 management was successfully conducted and the difference between the mean pre-intervention and postintervention scores in doctors, nurses, and other health care professionals was statistically very highly significant. Also, the feedback received from the participants who attended this training program was Excellent or Good in $92 \%$ to $94 \%$ of the responses. This showed that the training program was effective and thus achieved the objective of the in-situ simulation training program.

\section{Discussion}

Given the ongoing Coronavirus pandemic worldwide, the healthcare workers of the hospital need to be better trained in the in-situ simulation environment before handling a serious respiratory infection outbreak situation in reallife. The learning materials provided during the program, such as guidelines and patient information sheets were obtained from reliable and reputed sources, which gave confidence and clarity to the health care workers.
Simulation-based education is a recognized method for health care- professionals' education. It is increasingly being employed for teaching, learning, training, and assessment of health-care workers. Simulation-based education reduces the uneasiness of medical care experts by creating and refreshing their insight, abilities, and perspectives while removing unwarranted risk to patients. Teachers must be trained in simulation-based education to improve the efficiency of learners. ${ }^{14}$

In-situ simulation can also be used as a probe to provoke errors (Latent error detection) and to analyse through a simulated repeat of the event. ${ }^{15}$

In-situ simulations additionally furnished a way to enhance collaboration practices, for example, the utilization of assertive explanations, position clarity, the overall execution of common updating, improvement of a shared intellectual model, execution of autonomous two-fold checks of high-risk medications, and removing authority inclinations among group members. Participants expressed that the preparation program was compelling and did not disturb patient care. ${ }^{16}$

COVID-19 pandemic has caused a lot of human suffering in the past few months. Keeping the infectiousness of COVID-19 in mind, it is indispensable that adequate infection prevention and control practices are practiced in the health care setup.

Prevention of COVID-19 infection in health care professionals is very important for the protection of health workers themselves and also for the reason that they do not become the source of infection. Hence, training the doctors, nurses, and other health care workers becomes imperative. It is also beneficial to test the knowledge after the training session which shows whether the objectives of the training session were achieved.

In this study, the difference between the mean preintervention and post-intervention scores in doctors, nurses, and other health care professionals was statistically very highly significant before and after the training 
Table 2 Participants' Feedback on Various Characteristics of the in-situ Simulation Program and the Response in Number Percentage

\begin{tabular}{|c|c|c|c|c|}
\hline & \multicolumn{4}{|c|}{ Participants Feedback $(n=50)$ Number $(\%)$} \\
\hline & Excellent & Good & Satisfied & Not Satisfied \\
\hline The relevance of the topic on a need basis & $19(38)$ & $28(56)$ & $2(4)$ & I (2) \\
\hline Gained adequate expertise & $12(24)$ & $28(56)$ & $9(18)$ & I (2) \\
\hline Increased confidence over attitude & $18(36)$ & $28(56)$ & $4(8)$ & $0(0)$ \\
\hline Adequate materials to follow protocol & $17(34)$ & $29(58)$ & $4(8)$ & $0(0)$ \\
\hline
\end{tabular}

session. Thus, this training session in the form of a simulated situation of respiratory disease outbreak management with special reference to COVID-19 was immensely helpful in training the doctors, nurses, and other health care workers in donning and doffing of personal protective equipment (PPE) and situation management in screening area of the outpatient department. They were also trained regarding hand hygiene, respiratory hygiene, and infection control practices.

The participants were happy with the learning materials which provided them related information to work effectively in real situations. They watched the various steps demonstrated in the training and then practiced further by doing themselves after the program. We have received significant positive feedback from the participants (Table 2).

The participants' feedback revealed that they obtained confidence after attending the program and got their doubts on technical issues clarified related to infection prevention control and guidelines.

The principal contention for simulation-based teaching (whether in-situ or in the laboratory environment) is that it gives a protected place to gain from errors. ${ }^{17}$

The in-situ simulation is an active technique not only a preparation situation sanctioned at the clinical ward followed by a static questioning. Doctors' and nurses' contribution to protection attitudes, also, safety cultures are not independent of doctors and nurse's information and abilities about patient wellbeing and group correspondence. ${ }^{18}$

In-situ simulation program can be critical for development in crisis staff's wellbeing and cooperation perspectives that may relate with more positive patient protection culture. ${ }^{19}$

Hence, frequent training, making training modules, and updating the training modules depending upon the sudden emergence of infection outbreaks are mandatory in inpatient and public care settings.

The utilization of "event set" triggers and distracters prompt a group collaboration and stresses interdisciplinary group work. Subsequently, the loyalty and sharpness of the simulation is expanded and makes the adequate opportunity for group members to commit errors and permits spectators to acknowledge where cycles separate. This gives a rich source to deal with progress. ${ }^{20}$

According to Weller et al, simulation-based education adds to the learning of medical students and physicians in decision-making, interprofessional correspondence, correspondence with patients, collaboration, and clinical and procedural abilities. They explained that learning experience with a genuine patient is key for getting clinical aptitude, yet simulation gives an occasion to rehearse in limiting odds of blunder. They further clarified that simulation-based education guarantees confidence and ability to medical students before they start dealing with real situations. Simulation-based education requires educational plan incorporation, satisfactory framework, and prepared workforce clarifying it. $^{21}$

However, various limitations were observed by educators. Patterson et al, in a post-intervention study, highlighted that $77 \%$ of the healthcare workers revealed practically zero clinical effect despite running in-situ simulations for over 1 year ${ }^{22}$. The healthcare workers trained in this exercise need to be transferred immediately to attend to the patients when the crisis occurs. ${ }^{23}$ Hence, there is a need to have more manpower to balance the workload in the hospital.

\section{Limitation}

A comparison between the participant's and the control group's effectiveness in handling acute respiratory infection outbreaks might have given additional information in the analysis of the effectiveness of the in-situ simulation program.

\section{Conclusion}

To manage the crisis of viral pandemic outbreak worldwide, there is a need for front-line workers of a hospital to get knowledge as well as skill in the screening of patient, 
diagnosis, and treating the patients. Self-protection from the transmission of infection during the various procedures of infection outbreak management is equally important, if not more.

The in-situ simulation model is a training method and it is similar to a functional Mock Drill to improve the job performance of health care professionals. It helps by updating and standardizing essential information as per the guidelines of competent authority, knowledge, and skills to avoid the spread of infections to healthcare workers, patients, communities and to care for the patient.

To measure the readiness and effectiveness of an emergency response plan with simulated health care personnel and patient, an in-situ simulation is one of the possible way closer to the real-life scenario. Hence, it is a useful tool to assess disaster preparedness against any type of outbreak.

\section{Strengths of the Study}

The follow-up assessment of the knowledge gained and implementation in routine practice was made in the form of monitoring. Checklist (Supplementary material) of do's and don'ts in the actual COVID-19 patient management started in the hospital from the beginning of March 2020, especially within the laboratory diagnostic team and infection prevention control management team.

\section{Abbreviations}

HCW, health care workers; WHO, World Health Organization; AIIMS, All India Institute of Medical Sciences.

\section{Data Sharing Statement}

The datasets used and analysed during this study are available from the corresponding author upon reasonable request.

\section{Ethics Approval and Informed Consent}

The Ethical clearance for the study was obtained from the Institutional Ethical Committee (IEC) and the Informed consent was taken from all participants.

\section{Consent for Publication}

Consent to participate in the study and to allow data to be published was obtained from all study participants.

\section{Acknowledgment}

Prof. Dr. T.S. Ravikumar M.S., FRCS(Edinburgh), FRCS (Canada)., FACS, President, All India Institute of Medical Sciences (AIIMS), Mangalagiri (A.P), India. Prof. Dr. Mukesh Tripathi M.D., FAMS, Director \& C.E.O, AIIMS, Mangalagiri. Dr. Manuj Sarkar, Physician, and the participant doctors, nurses, laboratory technicians of All India Institute of Medical Sciences, Mangalagiri, Andhra Pradesh, India.

\section{Funding}

There is no funding to report.

\section{Disclosure}

The authors declare that they have no conflicts of interest for this work.

\section{References}

1. Infection prevention and control during health care when novel coronavirus (2019-nCoV) infection is suspected Interim guidance January 2020. WHO/2019-nCoV/IPC/v2020.

2. Gurav YK, Potdar V, Abraham P. Specimen collection, packaging, and transport guidelines for 2019 novel coronavirus (2019-nCoV). ICMR-NIV/2019-nCoV/Specimens_01.

3. Detailed Guidelines for Infection Prevention Control for Suspected Cases of 2019- nCoV Acute Respiratory Disease. Ministry of Health and Family Welfare, Government of India; 2019. Available from: https://ncdc.gov.in/WriteReadData/1892s/53243150651583746411. pdf. Accessed 18 February 2020.

4. Kozlowski SWJ, Chao GT, Grand JA, Braun MT, Kuljanin G. Advancing multilevel research design: capturing the dynamics of emergence. Organ Res Methods. 2013;16(4):581-615. doi:10.1177/ 1094428113493119

5. Al-Elq AH. Simulation-based medical teaching and learning. J Family Community Med. 2010;17(1):35-40. doi:10.4103/1319-1683.68787

6. Lighthall GK, Poon T, Harrison TK. Using in situ simulation to improve in-hospital cardiopulmonary resuscitation. Jt Comm J Qual Patient Saf. 2010;36:209-216. doi:10.1016/s1553-7250(10)36034-x

7. Geis GL, Pio B, Pendergrass TL, et al. Simulation to assess the safety of new healthcare teams and new facilities. Simul Healthc. 2011;6 (3):125-133. doi:10.1097/SIH.0b013e31820dff30

8. Shrestha R, Shrestha AP, Shrestha SK, et al. Interdisciplinary in situ simulation-based medical education in the emergency department of a teaching hospital in Nepal. Int J Emerg Med. 2019;12(1):19. doi:10.1186/s12245-019-0235-x

9. Rosen MA, Hunt EA, Pronovost PJ, Federowicz MA, Weaver SJ. In situ simulation in continuing education for the health care professions: a systematic review. J Contin Educ Health Prof. 2012;30 (2):78-88. doi:10.1002/chp

10. World Health Organization Regional Office for Africa Brazzaville • 2015. Protocol for the Investigation of Acute Respiratory Illness Outbreaks of Unknown Etiology. (C) WHO Regional Office for Africa; 2016.

11. Draft as of 3 February 2020; 2019 Novel Coronavirus (2019-nCoV): strategic preparedness and response plan. Available from:https:// www.who.int/publications-detail/global-surveillance-forhumaninfection-with-novel-coronavirus-(2019-ncov). Accessed May 12, 2021. 
12. SOP on Mock exercise. National disaster response force ministry of home affairs. Government of India. Available from: www.ndrfanded. gov.in. Accessed May 12, 2021.

13. WHO/COVID-19/community actions/2020 critical preparedness, readiness and response actions for COVID-19, interim GUIDANCE 16 March 2020.

14. Piryani RM, Piryani S, Shrestha U, et al. Simulation-based education workshop: perceptions of participants. Adv Med Educ Pract. 2019;10:547. doi:10.2147/AMEP.S204816

15. Hunt EA, Shilkofski NA, Stavroudis TA, Nelson KL. Simulation translation to improved learn performance. Anaesthesiol Clin. 2007;25(2):301-319. doi:10.1016/j.anclin.2007.03.004

16. Wheeler DS, Geis G, Mack EH, LeMaster T, Patterson MD. Highreliability emergency response teams in the hospital: improving quality and safety using in situ simulation training. BMJ Qual Saf. 2013;22:507-514. doi:10.1136/bmjqs-2012-000931

17. Ziv A, Wolpe PR, Small SD, Glick S. Simulation-based medical education: an ethical imperative. Acad Med. 2003;78(8):783-788. doi:10.1097/01.SIH.0000242724.08501.63

18. Weaver SJ, Lubomksi LH, Wilson RF, Pfoh ER, Martinez KA, Dy SM. Promoting a culture of safety as a patient safety strategy. Ann Intern Med. 2013;158(5_Part_2):369-374. doi:10.7326/00034819-158-5-201303051-00002
19. Paltved C, Bjerregaard AT, Krogh K, Pedersen JJ, Musaeus P. Designing in situ simulation in the emergency department: evaluating safety attitudes amongst physicians and nurses. Adv Simul. 2017;2 (1):4. doi:10.1186/s41077-017-0037-2

20. Davis SE, Miller K, Riley W, Hansen H. Reducing patient harm through interdisciplinary team training with in situ simulation. Proceedings of the Improving Patient Safety Conference; 2008.

21. Weller JM, Nestel D, Marshall SD, Brooks PM, Conn JJ. Simulation in clinical teaching and learning. Med J Aust. 2012;196(9):594. doi:10.5694/mja10.11474

22. Patterson MD, Geis GL, Falcone RA, et al. In situ simulation: detection of safety threats and teamwork training in a high risk emergency department. BMJ Qual Saf. 2013;22(6):468-477. doi:10.1136/bmjqs-2012-000942

23. Abrahamson SD, Canzian S, Brunet F, et al. Using simulation for training and to change protocol during the outbreak of severe acute respiratory syndrome. Crit Care. 2006;10(1):R3. doi:10.1186/cc3916

\section{Publish your work in this journal}

Advances in Medical Education and Practice is an international, peerreviewed, open access journal that aims to present and publish research on Medical Education covering medical, dental, nursing and allied health care professional education. The journal covers undergraduate education, postgraduate training and continuing medical education including emerging trends and innovative models linking education, research, and health care services. The manuscript management system is completely online and includes a very quick and fair peer-review system. Visit http://www.dovepress.com/testimonials.php to read real quotes from published authors. 